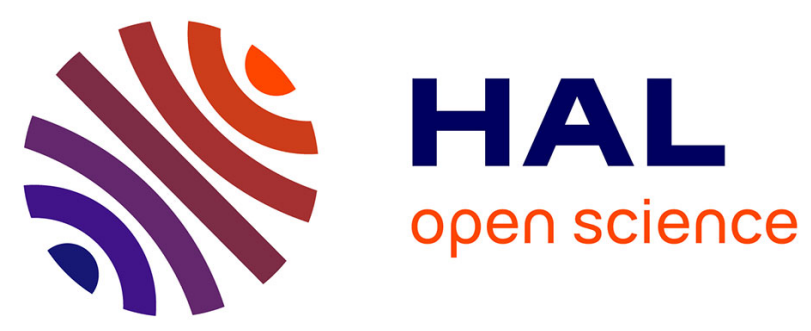

\title{
Production de champs magnétiques intenses transitoires supérieurs a 250 teslas par un montage de compression de flux magnétique ouvert a la transmission optique
}

\author{
J. Besançon, M. Guillot, F. Herlach
}

\section{- To cite this version:}

J. Besançon, M. Guillot, F. Herlach. Production de champs magnétiques intenses transitoires supérieurs a 250 teslas par un montage de compression de flux magnétique ouvert a la transmission optique. Revue de Physique Appliquée, 1977, 12 (4), pp.573-579. 10.1051/rphysap:01977001204057300 . jpa-00244211

\author{
HAL Id: jpa-00244211 \\ https://hal.science/jpa-00244211
}

Submitted on 1 Jan 1977

HAL is a multi-disciplinary open access archive for the deposit and dissemination of scientific research documents, whether they are published or not. The documents may come from teaching and research institutions in France or abroad, or from public or private research centers.
L'archive ouverte pluridisciplinaire HAL, est destinée au dépôt et à la diffusion de documents scientifiques de niveau recherche, publiés ou non, émanant des établissements d'enseignement et de recherche français ou étrangers, des laboratoires publics ou privés. 
Classification

Physics Abstracts

0.665

\title{
PRODUCTION DE CHAMPS MAGNÉTIQUES INTENSES TRANSITOIRES SUPÉRIEURS A 250 TESLAS PAR UN MONTAGE DE COMPRESSION DE FLUX MAGNÉTIQUE OUVERT A LA TRANSMISSION OPTIQUE $\left(^{*}\right)$
}

\author{
J. BESANÇON (**) \\ Centre d'Etudes de Vaujours (CEA/DAM), BP No 7, 93270 Sevran, France
}

\section{GUILLOT}

C. N. R. S., Laboratoire`de Magnétisme, 166 X-38042 Grenoble Cedex, France

F. HERLACH $(* * *)$

Katholieke Universiteit, Leuven, Belgique

(Reçu le 14 octobre 1976, révisé le 6 janvier 1977, accepté le 11 janvier 1977)

Résumé. - Description d'un montage de compression de champ magnétique au moyen d'explosif chimique permettant de produire des champs de l'ordre de 300 teslas. L'étude détonique d'un implosoir réalisé simplement par découpage puis assemblage de feuilles d'explosif commercial, est présentée. La cavité mégagauss ouverte à la transmission permet des réglages optiques plus faciles.

Abstract. - In this report we present a magnetic flux compression system using explosivedriven implosion of a metallic tube. An explosive device derived from the top hat configuration is described ; the charge and the initiation sheet are obtained by using commercially available explosive sheet. The cylindrical megagauss cavity is open to the transmission light beam. Fields up to 300 teslas are obtained (English translation available).

1. Introduction. - Lorsqu'il s'agit d'atteindre des champs magnétiques intenses transitoires supérieurs à $200 \mathrm{~T}$, la compression de flux magnétique au moyen d'explosif chimique reste pratiquement la seule technique utilisée. La première de ces expériences (1 $430 \mathrm{~T})$, effectuée par le groupe de Los Alamos, date de 1960 [1] ; le compte rendu de la Conférence de Frascati (1965) [2] constitue une excellente revue des activités développées à la suite des travaux de l'équipe de C. M. Fowler [1]. Le flux magnétique primaire produit par la décharge d'une batterie de condensateurs est piégé par un tube métallique, le liner, qui est ensuite mis en vitesse par l'onde de détonation et implosé sur son axe sous l'action de gaz en détente provenant de l'explosif. Différentes dispositions relatives de l'explosif, de la bobine de champ et du liner ont été présentées (Fig. 1). Parmi elles, le montage top hat (Fig. 1a), [2] constitue, notamment par sa simplicité, son coût modique et sa fiabilité, une solution élégante $[3,4]$.

(*) English translation available.

(**) Nouvelle adresse : Centre d'Etudes de Limeil, B. P. 27, 94 Villeneuve-St-Georges, France.

$(* * *)$ Participation aux travaux effectués, au cours d'un stage comme collaborateur étranger au Centre d'Etudes Nucléaires de Grenoble (Laboratoire des Applications Spéciales de la Physique) B. P. 85 X-38041 Grenoble Cedex.

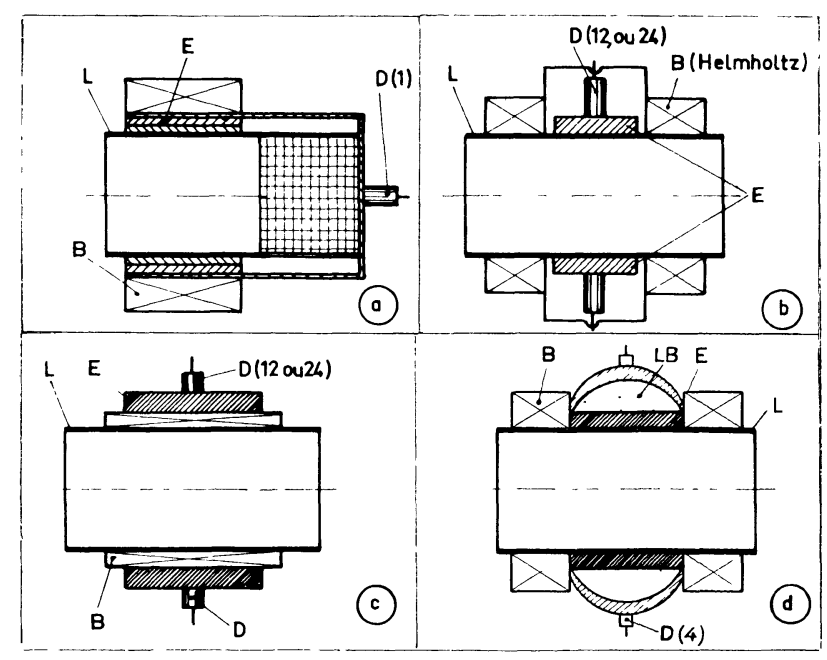

FIG. 1. - Représentation schématique des différentes configurations de compression de champ magnétique : $\mathbf{B}$ : bobine de production du champ primaire ; $\mathrm{L}:$ liner $; \mathrm{E}$ : explosif ; $\mathrm{D}$ : détonateur (le nombre entre parenthèses représente le nombre de détonateurs utilisés); LB : lentilles en bi-explosif.

[Schematical representation of different configurations for the compression of magnetic fields. B : coil for the generation of the initial field, $\mathrm{L}$ : liner, $\mathrm{E}$ : explosive, $\mathrm{D}$ : detonator (the number given in brackets is the total number of detonators), LB : explosive detonation wave generators (lenses made with explosives of different detonation speed). ] 

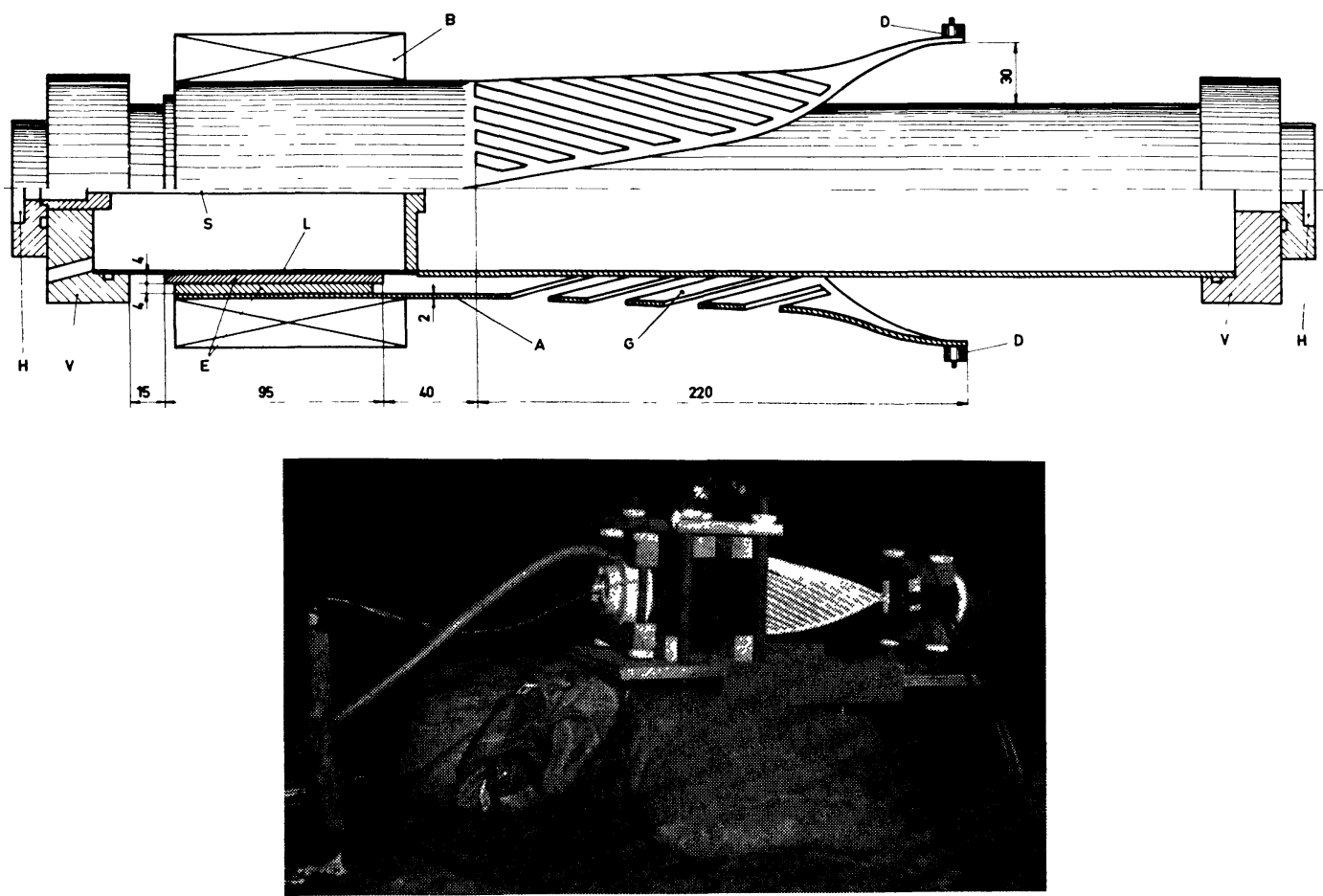

Fig. 2. - Dessin côté du système de compression de flux et photographie de l'engin avant le tir : G : grille ou générateur d'onde circulaire; $A$ : couche d'amorçage ; $B$ : bobine champ primaire ; $D$ : détonateur ; E : explosif de renforcement ; $\mathbf{H}$ : hublots optiques ; L : liner ; S : sondes de mesure de champ ;

$\mathrm{V}$ : bouchon assurant l'étanchéité (vide compris entre $10^{-2}$ et $10^{-3}$ torr).

[Scale drawing of the flux compression device and a photograph of the assembled device before the shot. $\mathbf{G}:$ grid (i. e. circular wave generator), $\mathbf{A}:$ transmission sheet, $\mathrm{B}:$ coil for the initial field, $\mathrm{D}:$ detonator, $\mathrm{E}$ : main explosive charge, $\mathrm{H}$ : optical windows, $\mathrm{L}:$ liner, $\mathrm{S}$ : probes for the field measurement, $\mathrm{V}:$ lid forming a vacuum chamber (vacuum in the range $10^{-2}-10^{-3}$ torr).]

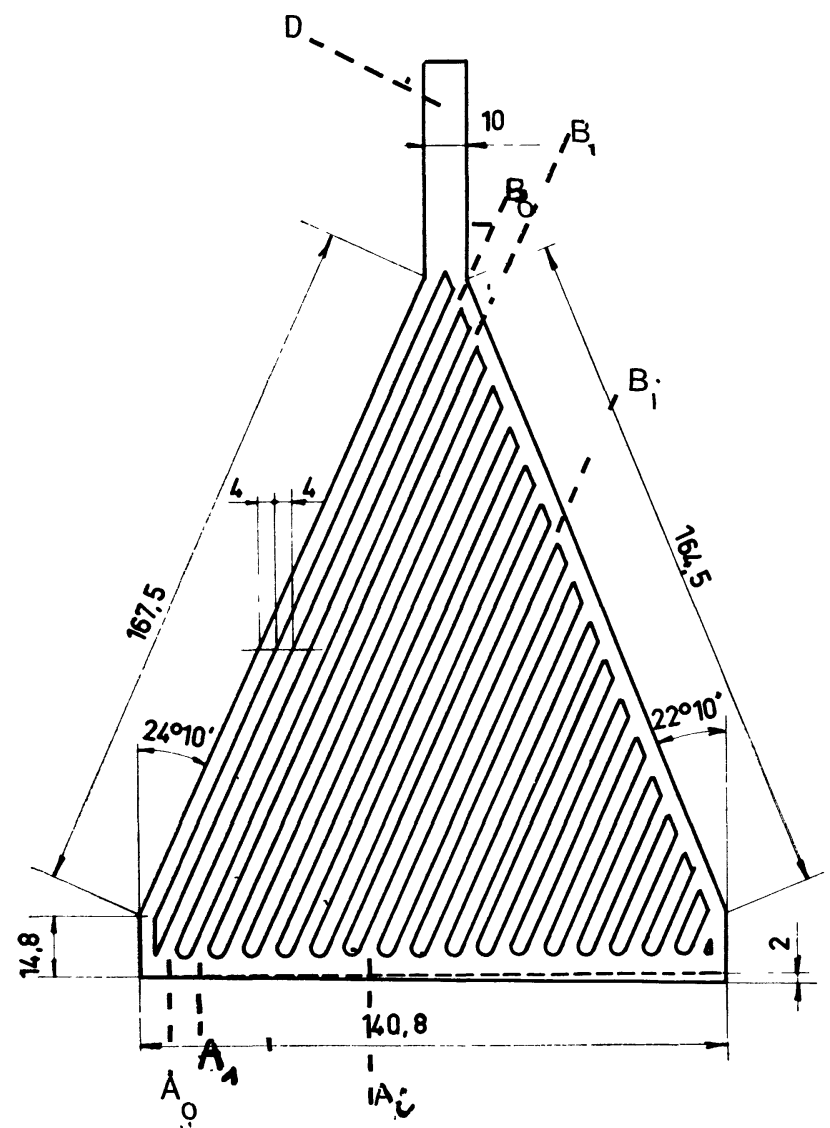

(a)



(b)

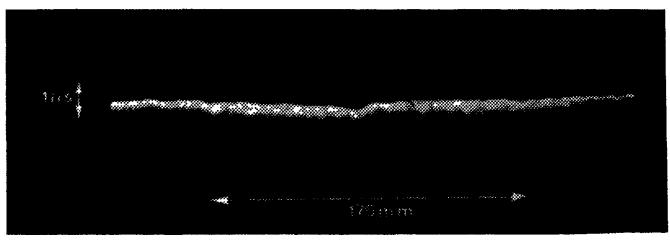

(c)

Fig. 3. - Schéma de la grille utilisée pour l'étude en géométrie plane; profils de l'onde de détonation observée à la caméra à fente pour un générateur (b) et deux générateurs placés côte à côte (c).

[Schematic drawing of the grid-shaped line wave generator as used for studies in plane geometry; detonation wave profiles observed by means of a streak camera for one generator (b) and two generators joint side by side (c).] 
Lorsque le but recherché n'est pas seulement l'obtention de champ intense mais en plus l'utilisation de ce champ à l'étude de phénomènes physiques, il apparaît que les techniques magnétooptiques semblent les seules bien adaptées aux conditions particulières de ces expériences [5,6]. La configuration top hat présente alors l'inconvénient d'offrir une cavité mégagauss $\left({ }^{1}\right)$ fermée à une extrémité, ce qui nécessite un miroir de renvoi dont le réglage est toujours délicat; comme de plus, les radiations lumineuses utilisées, peuvent être hors du visible (infrarouge notamment), la supériorité d'un montage ouvert à la transmission devient certaine. Dans les dispositifs ouverts proposés jusqu'à ce jour [4], la charge explosive est généralement un cylindre creusé et soigneusement usiné aux dimensions du liner ou de la bobine; la mise en détonation s'effectue soit par une couronne de 12 ou 24 détonateurs, également répartis sur le diamètre extérieur de la charge (Fig. $1 b$, 1c), soit par des générateurs d'onde cylindrique (lentille

(1) Le système d'unités est le système M. K. S. A. Cependant, pour rester fidèle à la tradition, nous parlerons de champ mégagauss $\left(1 \mathrm{~T}=10^{4} \mathrm{G}\right)$. en bi-explosif : Fig. 1d) dont la réalisation demande une précision meilleure que $0,1 \mathrm{~mm}$ [7]. De telles solutions imposent des ateliers spécialement équipés, et ce n'est pas notre cas dans les laboratoires grenoblois, de plus, elles s'adaptent mal à l'emploi de feuilles d'explosif que l'expérimentateur découpe aux dimensions voulues avant de les coller puis de les enrouler sur le liner, sur les lieux mêmes de l'expérience.

Dans cet article, nous présentons un implosoir, c'est-à-dire un générateur d'onde cylindrique convergente réalisé à partir d'explosif commercial : le Formex $\mathrm{F}_{4} \mathrm{HV}$, fabriqué par la Société Nationale des Poudres et explosifs (SNPE). Le Formex contient $89 \%$ de penthrite ; le liant est de la poudre de caoutchouc naturel; ce qui confère aux feuilles dont l'épaisseur est 1, 2 ou $4 \mathrm{~mm}$ une certaine souplesse. Les caractéristiques détoniques sont les suivantes: la vitesse de détonation est $6940 \pm 150 \mathrm{~ms}^{-1}$ (mesurée pour une feuille d'épaisseur $2 \mathrm{~mm}$ ), la densité de chargement égale à $1300 \mathrm{kgm}^{-3}$ et la pression au point Chapman-Jouguet de l'ordre de 12 GPa (120 kbars).

2. Description de l'implosoir. - Rappelons tout d'abord les principales caractéristiques du montage



Fig. 4. - Ensemble des deux générateurs d'onde linéaire ou grilles, prolongé par la couche de stabilisation (40 $\mathrm{mm})$. [Assembly of two grid-shapened line wave generators, extended by the stabilization sheet of $40 \mathrm{~mm}$ length.] 
[6]; le liner, en acier inoxydable $\left({ }^{2}\right)$ a un diamètre extérieur de l'ordre de $80 \mathrm{~mm}$, une épaisseur de $1,5 \mathrm{~mm}$, une longueur utile de $120 \mathrm{~mm}$; la bobine de champ primaire $(6,5 \mathrm{~T}$ pour un temps de montée de l'ordre de la milliseconde) a un diamètre intérieur de l'ordre de $100 \mathrm{~mm}$, diamètre extérieur de l'ordre de $140 \mathrm{~mm}$ (compte tenu de l'isolement et du renforcement mécanique), une longueur de $140 \mathrm{~mm}$ (Fig. 2).

La charge explosive de renforcement est formée de deux couches concentriques de $4 \mathrm{~mm}$ d'épaisseur, respectivement de 95 et $85 \mathrm{~mm}$ de longueur (un tel décalage diminue les risques de cisaillement du liner au moment de sa mise en vitesse). Ces couches sont en Formex $\quad \mathrm{F}_{4} \mathrm{HV}$; l'explosif Detasheet à $90 \%$ de penthrite, de fabrication américaine (Dupont de Nemours) et de performance analogue au Formex, a été aussi retenu pour cet usage. Sur le cylindre ainsi obtenu est enroulée la couche d'amorçage qui se prolonge jusqu'aux deux détonateurs par un dispositif de mise en forme circulaire de l'onde de détonation.

La figure $3 a$ qui représente un développement plan partiel de ce dispositif en forme de grille, permet de comprendre l'idée de base; en partant d'un point d'amorçage $\mathrm{D}$ (correspondant au centre du détona-

(2) Nuance Z 3 CN 18/10.



Statique teur), l'onde de détonation atteint les points $A_{0}$, $A_{1}, \ldots, A_{i}$ aux mêmes instants si les chemins $D B_{0} A_{0}$, $\mathrm{DB}_{1} \mathrm{~A}_{1}, \ldots, \mathrm{DB}_{i} \mathrm{~A}_{i}$ sont égaux. Expérimen`alement, le profil de l'onde est visualisé au moyen d'une caméra à fente ; une lame d'air de faible épaisseur $(0,1 \mathrm{~mm}$ environ), placée à l'extrémité de la grille perpendiculairement au plan de l'onde, devient luminescente au passage de celle-ci. L'expérience montre que chaque bande de la grille donne naissance à une ondele'te de faible rayon de courbure (Fig. $3 b$ ) ; l'ensemble des bandes engendre un front d'onde ondulée dont les défauts en temps sont de l'ordre de 200 ns. Un décalage d'un côté à l'autre de la grille est rattrapé par un léger biseautage $(2 \mathrm{~mm})$ du bord inférieur de l'explosif. Dans les conditions géométriques définies par la figure $3 a$, tous les points détonent en phase avec un écart dans le temps effectivement inférieur à $200 \mathrm{~ns}$ soit dans l'espace 1,4 mm (Fig. $3 b$ ). La figure $3 c$ montre que cet écart n'est pas notablement modifié lorsque deux grilles sont placées côte à côte.

Il est important de noter que le découpage de la grille doit être effectué avec soin ; en particulier, les coupures doivent être très nettes; tous les copeaux sont à éliminer pour ne pas provoquer des amorçages entre bandes; le découpage au moyen d'un scalpel chirurgical, dans l'épure d'un gabarit, donne des résul-

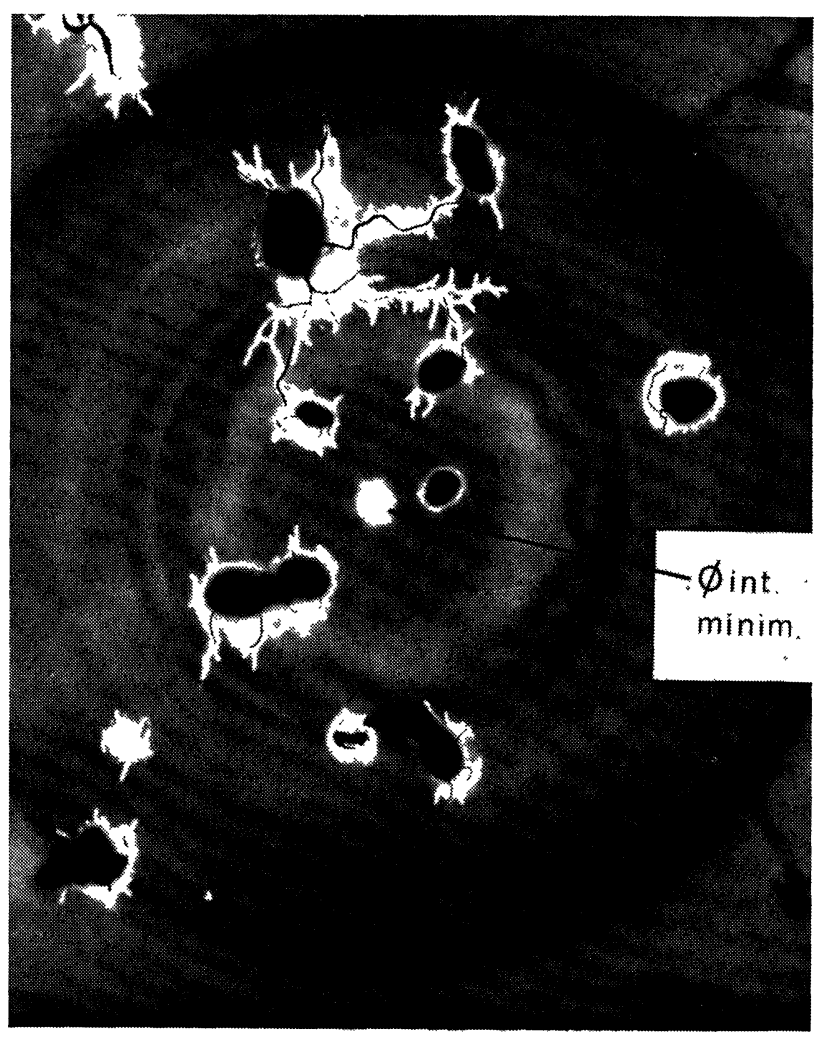

$t=60.1 \mu s$

FIG. $5 a$

FIG. 5. - Etude de la compression cylindrique au moyen de radiographie-éclair : dans l'axe (a) et latéralement (b). 'Study of the cylindrical implosion by means of flash x-ray photography : viewed along the axis (a) and laterally (b).] 


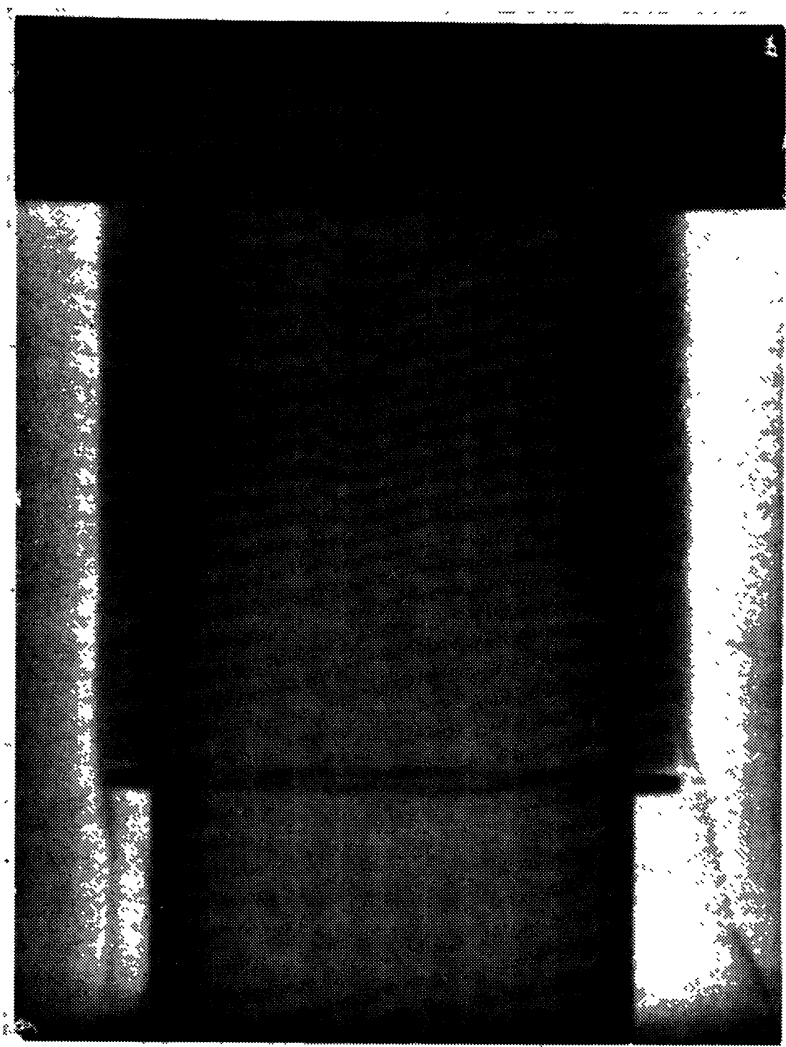

Statique

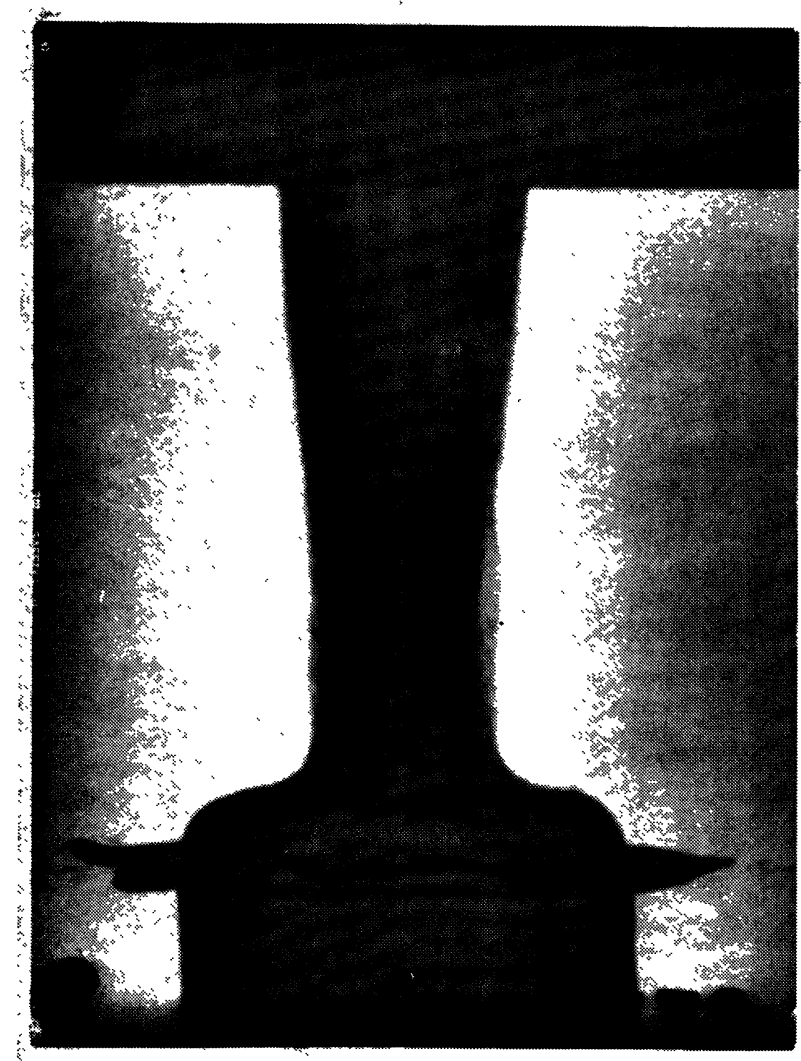

$t=61,3 \mu \mathrm{s}$

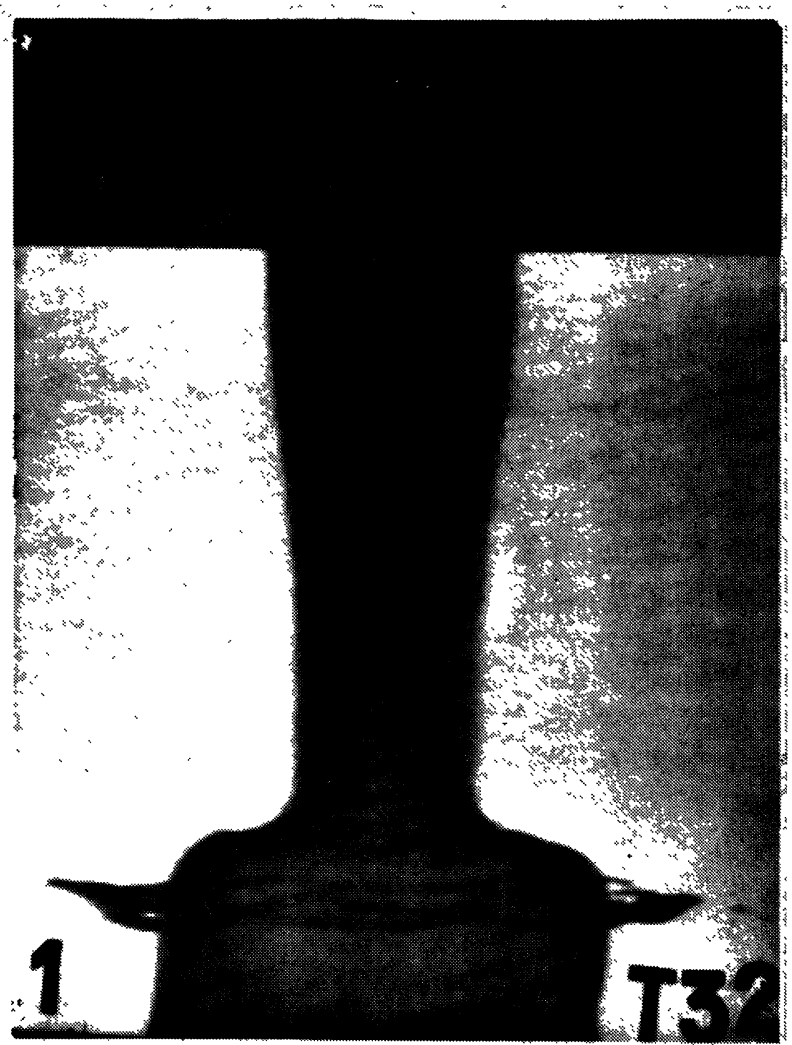

$\mathrm{t}=60.2 \mu \mathrm{s}$

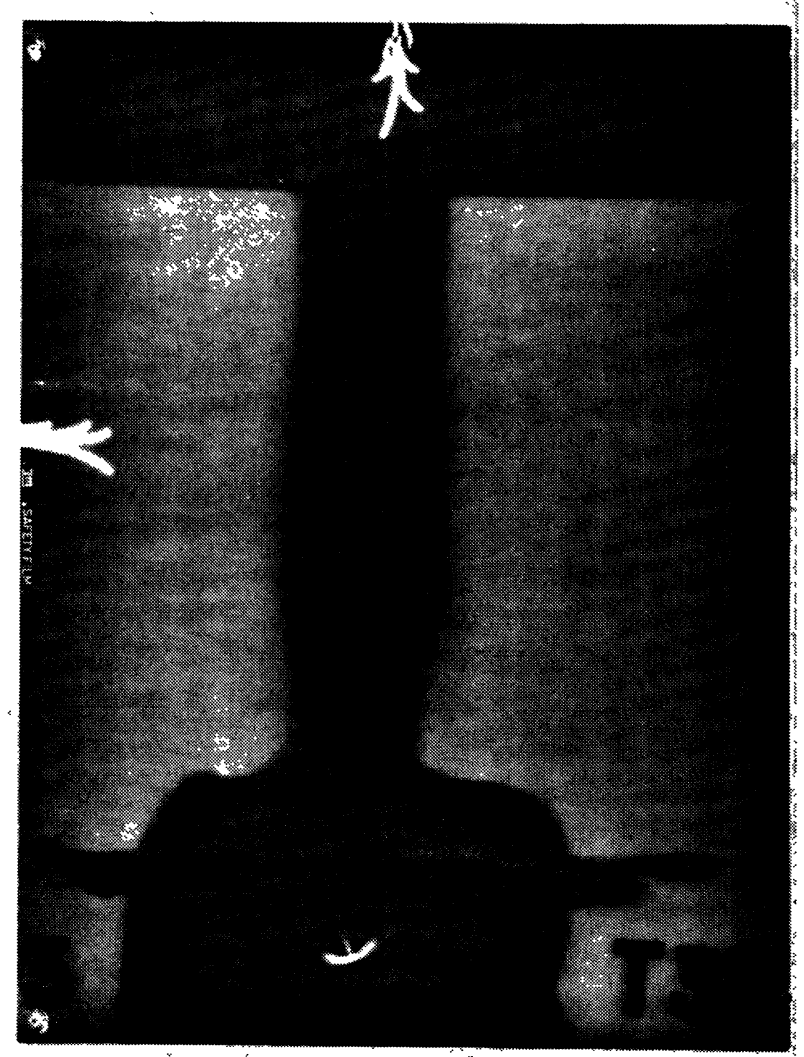

$t=70 \mu \mathrm{s}$

FIG. $5 b$ 
tats satisfaisants. Le temps nécessaire au découpage des grilles et au collage des différentes couches d'explosif est de l'ordre d'une quinzaine d'heures par un technicien qualifié. Dans ces conditions, l'onde de détonation dans la couche d'amorçage présente en coupe un profil pratiquement plan, ce qui est très recommandé pour éviter des instabilités pendant l'implosion cylindrique.

Lorsqu'il s'agit de passer à la géométrie cylindrique, deux difficultés se présentent. La première est liée à la nature même du Formex qui, lors de l'enroulement, peut être le siège d'amorces de cassure qui provoquent des discontinuités dans l'onde de détonation. A cet égard, la souplesse et la plasticité du Formex sont jugées satisfaisantes quand ce matériau explosif est utilisé frais. La seconde concerne la jonction entre les bords des grilles ; les dimensions de la grille (Fig. 3a), comparées à celles du liner impliquent l'utilisation de deux grilles (notons que le profil d'onde obtenue par une grille de dimensions doubles de celles de la figure 3 a présenté des défauts nettement supérieurs à 200 ns ; la solution d'une grille à grand rapport d'homothétie (2) a été définitivement abandonnée). Les raccords entre les grilles doivent être effectués en conservant à la couche d'amorçage la meilleure symétrie possible. Après différents essais, nous avons adopté la solution représenté en figure 4, obtenue en augmentant seulement de $7 \%$ les dimensions de la grille initiale et en conservant l'inclinaison des bandes. De plus, pour permettre à l'onde de détonation d'atteindre un régime stable et de présenter un profil linéaire, chaque grille est prolongée d'une longueur de Formex plein égal à $40 \mathrm{~mm}$.

La compression du liner suivant son axe a été observée aux rayons $X$ (la figure $5 a$ donne un exemple des vues obtenues). On constate que la compression du tube métallique, sans être parfaite, est cependant régulière bien que le raccordement des deux grilles d'amorçage entraîne une légère dissymétrie qui se conserve jusqu'à la focalisation.

L'onde de détonation initiée dans la couche d'amorçage se déplace parallèlement à l'axe du liner et, lorsqu'elle atteint les couches de renforcement, provoque leur amorçage. Le relèvement cylindrique du liner a été observé latéralement par trois éclairs successifs de rayons $X(600 \mathrm{keV})$, déclenchés aux temps $60,2 \mu \mathrm{s}$, $61,3 \mu$ s et $70 \mu$ s après la mise à feu. La figure $5 b$ donne des exemples de radiographie obtenue; la zone dans laquelle la compression est la plus rapide et qui peut être considérée comme cylindrique est située à $40 \mathrm{~mm}$ de l'extrémité du cylindre de renforcement (figure 6 : position des sondes).

3. Production de champs intenses. - Les résultats, ci-dessus développés, sont obtenus dans le laboratoire $\mathrm{du}$ Fort des Quatre-Seigneurs de l'agglomération grenobloise (6) et sont le fruit d'une collaboration entre le C. E. A. (Le Laboratoire des Applications Spéciales
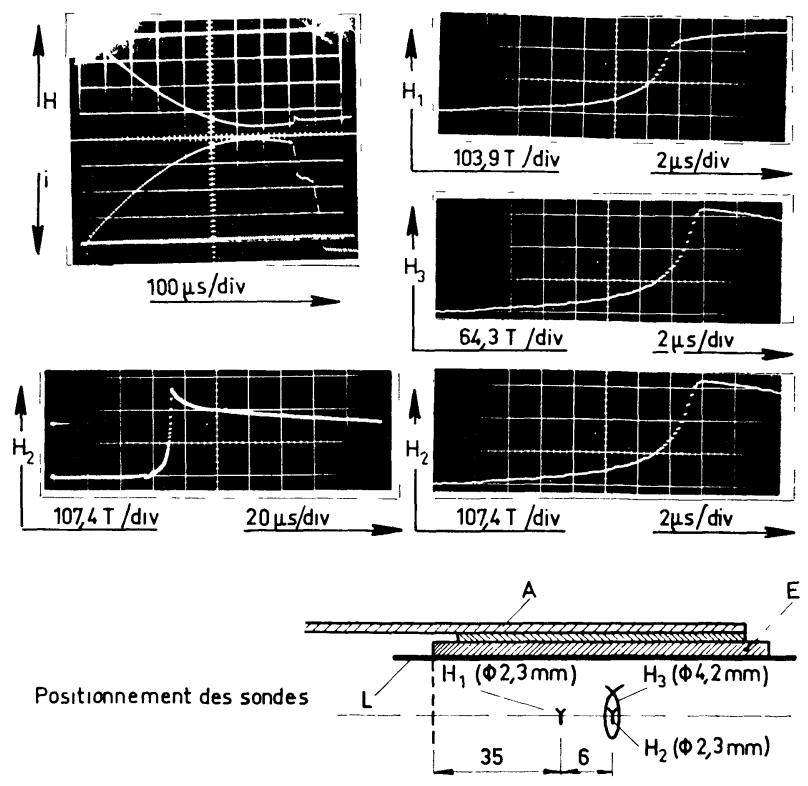

FIG. 6. - Oscillogrammes. La chronométrie de l'expérience est la suivante : Origine : déclenchement du banc de condensateurs ; $+800 \mu \mathrm{s}:$ mise à feu des deux détonateurs; les sondes de mesure sont détruites au temps $+870 \mu$ s. Sur la photographie en haut à gauche, les sondes de mesure du champ primaire et du courant de décharge ne sont pas étalonnées avec précision ; des expériences préliminaires montrent que le maximum du champ primaire est $6,3 \mathrm{~T}$. Le positionnement des sondes est déterminé par rapport au liner (Fig. 2).

[Oscilloscope records. The timing of the experiment is as follows. Origin : trigger of the capacitor bank, $+800 \mu \mathrm{s}:$ firing pulse to the detonators, the probes are destroyed at $+870 \mu \mathrm{s}$. The probes for the initial field and the current (left on top of the page) were not precisely calibrated, the field value of $6.3 \mathrm{~T}$ was derived from preliminary experiments. The positioning of the probes is related to figure 2.]

de la Physique du Centre de Grenoble et le Centre de Vaujours) et le C. N. R. S. (Laboratoire de Magnétisme et Service National des Champs Intenses).

La bobine de champ primaire a un diamètre intérieur de $100 \mathrm{~mm}$; elle comprend 38 à 40 spires de méplat de cuivre $6,5 \times 3 \mathrm{~mm}$ (ou de méplat $3 \times 2,5 \mathrm{~mm}$ ) ; l'isolement et la rigidité mécanique sont assurés par une imprégnation d'araldite et un enrobage de tissu de verre. Avec la batterie de condensateurs de $43 \mathrm{~kJ}$ ( $2440 \mu \mathrm{F}$ sous $5 \mathrm{kV}$ ), le champ primaire atteint $6,3 \mathrm{~T}$ en un temps de montée de $800 \mu \mathrm{s}$. Les sondes de mesure de champ ont un diamètre extérieur $\varnothing$ de $2,2 \mathrm{~mm}$; elles sont introduites dans un tube de verre de $\varnothing=3,2$ et $4,3 \mathrm{~mm}$. La figure 6 montre différents oscillogrammes obtenus; le maximum du champ est égal à $275 \mathrm{~T}(2,75 \mathrm{MG})$. La figure 7 représente les variations dans le temps du champ magnétique obtenu à l'aide d'enregistreurs de phénomènes aléatoires pour les mêmes sondes que celles de la figure 6 [5]. Notons que les sondes de mesure sont détruites avant que le champ n'atteigne son maximum, c'est-à-dire avant le rebondissement (considéré comme l'instant où le champ commence à décroître 


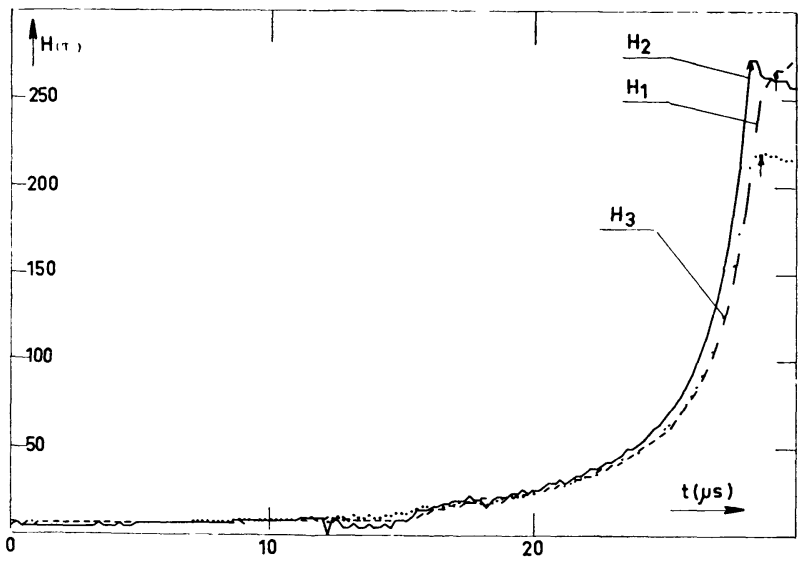

Fig. 7. - Variations dans le temps du champ magnétique obtenues à partir des sondes de la figure 6 et mesurées à l'aide d'enregistreurs de phénomènes aléatoires. Le zéro du temps de cette figure est arbitraire et n'a aucune relation avec la chronométrie de l'expérience.

[Magnetic field vs. time for the probes as indicated in figure 6, measured by means of transient digital recorders. The beginning of the time scale is not related to a fixed point in the timing of the experiment.]

par diffusion à travers le liner, avant que ce dernier n'ait abordé sa phase de décélération mécanique) [7].

4. Conclusion. - L'implosoir mis au point permet de préparer, avec des moyens relativement modestes, un montage de compression de flux donnant des champs pulsés dans la gamme des 3 mégagauss. Ceuxci sont sans doute susceptibles d'être augmentés en apportant notamment le plus grand soin à la préparation des charges explosives, en augmentant la valeur du champ primaire et en optimisant la nature du liner (cuivre, acier inoxydable) et son épaisseur afin de réduire les pertes de flux $[3,7]$. L'étude des propriétés magnéto-optiques des composés magnétiques à structure ordonnée (ferromagnétiques, antiferromagnétiques) s'effectue sur des échantillons dont l'épaisseur est de l'ordre de quelques dizaines de microns [5]. L'utilisation de lames plus épaisses se révèle en effet extrêmement difficile du fait de la très forte absorption dans le visible et le proche infrarouge des ions d'éléments de transition ( $\mathrm{Fe}, \mathrm{Ni}$, cobalt, terre rare). Dans ces conditions, l'uniformité du champ implosé ne doit pas être rigoureuse dans un volume important. En conclusion, il nous apparaît que notre dispositif présente des facilités d'alignement très importantes et doit permettre des mesures optiques (rotation Faraday, effet Zeemann) plus aisées dans la mesure où l'on se limite à des échantillons de faible longueur (au maximum de l'ordre du millimètre).

Note ajoutée aux épreuves : Durant la correction des épreuves nous prenons connaissance de l'article présenté par le groupe de Los Alamos à la Conférence de Pittsburgh [8] qui présente de nombreuses références sur le sujet encore que de récentes publications soviétiques ne soient pas mentionnées $[9,10]$.

Remerciements. - Nous remercions MM. Kehren et Thiberoz de la S. N. P. E. pour les nombreux entretiens qu'ils ont bien voulu nous accorder, MM. Signoret, Marlier, Bon Mardion (Centre d'Etudes Nucléaires de Grenoble) et M. Fouquoire (Centre d'Etudes de Vaujours) pour leur assistance technique et la D. R. M. E. qui subventionna en partie ces travaux. L'un des co-auteurs (F. H.) remercie le Laboratoire des Applications spéciales de la Physique pour son hospitalité.

\section{Bibliographie}

[1] Fowler, C. M., Garn, W., Caird, R. S., J. Appl. Phys., 31 (1960) 588

[2] Proceedings of the conference on megagauss magnetic field generation by explosives and related experiments; Frascati, Italie, sept. 1965, Euratom 2750 e.

[3] Herlach, F., Rep. on Prog. Phys. 31 (1968) 341.

[4] HeRlach, F., KNOEPFEL, H., Les champs magnétiques intenses, Conférences de Grenoble 1966 (édition du C. N. R. S.), $\mathrm{n}^{\circ} 166$.

[5] Guillot, M., Physique sous champs intenses, Conférence de Grenoble 1974 (édition du C. N. R. S.), no 242.
[6] Guillot, M., Revue Phys. Appl. 11 (1976) 647.

[7] Besancon, J.,Contribution à l'étude de la compression de champ magnétique intense par implosion d'un tube métallique. Thèse de Doctorat ès-sciences physiques, (Faculté des Sciences, Paris 1971), enregistrement au C. N. R. S., A. O. 5085 .

[8] Fowler, C. M. et al., I. E. E. E. Trans. Magnetics, MAG 12 (1976) 1018.

[9] PAVlovskil et al., J. E. T. P. Lett. 20 (1974) 256.

[10] Druzhinin, V. V. et al., J. E. T. P. Lett. 22 (1975) 130. 